\title{
CONDITIONAL LIMIT THEOREMS FOR BRANCHING PROCESSES ${ }^{1}$
}

\author{
LAJOS TAKÁCS \\ Case Western Reserve University \\ Cleveland, Ohio ${ }^{2}$
}

\begin{abstract}
Let $\{\xi(m), m=0,1,2, \ldots\}$ be a branching process in which each individual reproduces independently of the others and has probability $\boldsymbol{p}_{j}$ $(j=0,1,2, \ldots)$ of giving rise to $j$ descendants in the following generation. The random variable $\xi(m)$ is the number of individuals in the $m$ th generation. It is assumed that $\mathrm{P}\{\xi(0)=1\}=1$. Denote by $\rho$ the total progeny, $\mu$, the time of extinction, and $\tau$, the total number of ancestors of all the individuals in the process. This paper deals with the distributions of the random variables $\xi(m), \mu$ and $\tau$ under the condition that $\rho=n$ and determines the asymptotic behavior of these distributions in the case where $n \rightarrow \infty$ and $m \rightarrow \infty$ in such a way that $m / \sqrt{n}$ tends to a finite positive limit.
\end{abstract}

Key words: Critical branching processes, conditional limit theorems.

AMS (MOS) subject classifications: $\quad 60 \mathrm{~J} 80,60 \mathrm{~F} 05$.

\section{INTRODUCTION}

Let us suppose that in a population initially we have $i(i=1,2, \ldots)$ progenitors and in each generation each individual reproduces, independently of the others, and has probability $p_{j}$ $(j=0,1,2, \ldots)$ of giving rise to $j$ descendants in the following generation. Denote by $\xi(m)$ $(m=0,1,2, \ldots)$ the number of individuals in the $m$ th generation. We have $\mathbf{P}\{\xi(0)=i\}=1$.

Define

$$
\rho=\sum_{m \geq 0} \xi(m)
$$

that is, $\rho$ is the total number of individuals (total progeny) in the process (possibly $\rho=\infty$ ).

Let

\footnotetext{
${ }^{1}$ Received: August, 1991. Revised: September, 1991.

${ }^{2}$ Postal Address: 2410 Newbury Drive, Cleveland Heights, OH 44118
} 


$$
\mu=\sup \{m: \xi(m)>0\} .
$$

The random variable $\mu$ is interpreted as the time of extinction. If extinction never happens, then $\mu=\infty$. Furthermore, let

$$
\tau=\sum_{m \geq 0} m \xi(m)
$$

that is, $\tau$ is the total number of ancestors of all the individuals in the process (possibly $\tau=\infty$ ).

Our aim is to study the distributions of the random variables $\xi(m), \mu$ and $\tau$ under the condition that $\rho=n$ and determine the asymptotic behavior of these distributions if $n \rightarrow \infty$ and $m \rightarrow \infty$ in such a way that $m / \sqrt{n}$ tends to a finite positive limit. We shall assume that $\mathbf{P}\{\xi(0)=1\}=1$. The general case where $\mathbf{P}\{\xi(0)=i\}=1$ and $i \geq 1$ can be reduced to the particular case mentioned above.

\section{PRELIMINARIES}

The branching process $\{\xi(m), m \geq 0\}$ can also be interpreted in the following way: Let us suppose that in the time interval $(0, \infty)$ customers arrive at random at a counter and are served singly by one server. Let us assume that the server starts working at time $t=0$ and at that time $i(i=0,1, \ldots)$ customers are already waiting for service. Denote by $\nu_{r}(r=1,2, \ldots)$ the number of customers arriving at the counter during the $r$ th service time. Let us assume that $\nu_{1}, \nu_{2}, \ldots, \nu_{r}, \ldots$ are independent random variables for which

$$
\mathbf{P}\left\{\nu_{r}=j\right\}=p_{j}
$$

if $j=0,1,2, \ldots$ and $r=1,2, \ldots$. Write $N_{r}=\nu_{1}+\nu_{2}+\ldots+\nu_{r}$ for $r \geq 1$. Following D.G. Kendall [25] we say that the initial $i$ customers form the 0th generation. The customers (if any) arriving during the total service time of the initial $i$ customers form the 1st generation. Generally, the customers (if any) arriving during the total service time of the customers in the $(m-1)$ st generation form the $m$ th generation for $m=1,2, \ldots$ If $\xi(m)(m=0,1,2, \ldots)$ denotes the number of customers in the $m$ th generation, then $\{\xi(m), m \geq 0\}$ is the branching process defined in the Introduction.

The time of the server consists of alternating busy periods and idle periods. Denote by $\rho$ the number of customers served in the initial busy period. By the results of L. Takács [47], [49], we have

$$
\mathbf{P}\{\rho=n \mid \xi(0)=i\}=\frac{i}{n} \mathbf{P}\left\{N_{n}=n-i\right\}
$$


for $1 \leq i \leq n$. Formula (5) can also be interpreted as the probability that the total progeny in the branching process $\{\xi(m), m \geq 0\}$ is $n$ given that the number of progenitors is $i$. See also M. Dwass [14] and V.F. Kolchin [32] p. 104.

Let us introduce the generating function

$$
f(z)=\sum_{j=0}^{\infty} p_{j} z^{j}
$$

for $|z| \leq 1$. If $|w|<1$, then the equation

$$
z=w f(z)
$$

has exactly one root $z=g(w)$ in the unit disk $|z|<1$. By continuity we can extend the definition of $g(w)$ for $|w| \leq 1$ such that $|g(w)| \leq 1$ and $z=g(w)$ satisfies (7) for $|w| \leq 1$. By Lagrange's expansion we obtain that

$$
[g(w)]^{i}=i \sum_{n=i}^{\infty} \frac{w^{n}}{n} \mathbf{P}\left\{N_{n}=n-i\right\}
$$

if $i=1,2, \ldots$ and $|w| \leq 1$. Accordingly,

$$
\mathbf{E}\left\{w^{\rho} \mid \xi(0)=i\right\}=[g(w)]^{i}
$$

if $i=1,2, \ldots$ and $|w| \leq 1$.

Throughout this paper we make the following assumptions:

$$
\begin{gathered}
\sum_{j=0}^{\infty} p_{j}=1, \\
a=\sum_{j=0}^{\infty} j p_{j}=1, \\
\sigma^{2}=\sum_{j=0}^{\infty}(j-a)^{2} p_{j}
\end{gathered}
$$

is a finite positive number $(\sigma>0)$, and

$$
\operatorname{gcd}\left\{j: p_{j}>0\right\}=d .
$$

Then $f(1)=1, f^{\prime}(1)=a=1$ and $f^{\prime \prime}(1)=\sigma^{2}$ where the derivatives are left derivatives at $z=1$. Throughout this paper we assume that $\mathbf{P}\{\xi(0)=1\}=1$. If $\mathbf{P}\{\xi(0)=1\}=1$, then by (5)

$$
\mathbf{P}\{\rho=n\}=\mathbf{P}\left\{N_{n}=n-1\right\} / n
$$

for $n=1,2, \ldots$ Each possible value of $\rho$ has the form $n=s d+1$ where $s=0,1,2, \ldots$. We have $\mathbf{P}\{\rho<\infty\}=1$ and for any $\epsilon>0$, there exists an $N=N(\epsilon)$ such that 


$$
\left|\mathbf{P}\{\rho=n\}-\frac{d}{\sqrt{2 \pi \sigma^{2} n^{3}}}\right|<\frac{\epsilon}{\sqrt{n^{3}}}
$$

provided that $n=s d+1(s=0,1, \ldots)$ and $n>N(\epsilon)$. Since in (14) $N_{n}=\nu_{1}+\nu_{2}+\ldots+\nu_{n}$ is the sum of $n$ independent and identically distributed random variables with a finite positive variance $\sigma^{2}$, we can apply to $N_{n}$ the local central limit theorem of B.V. Gnedenko [18] to obtain (15). (See also B.V. Gnedenko and A.N. Kolmogorov [20] p. 233.)

By an Abelian theorem we obtain that

$$
g(w)=1-\sqrt{2(1-w) / \sigma^{2}}\left[1+\epsilon_{1}(w)\right]
$$

where $\epsilon_{1}(w) \rightarrow 0$ as $w \rightarrow 1(|w|<1)$, and

$$
\lim _{w \rightarrow 1} g^{\prime}(w) \sqrt{1-w}=1 / \sqrt{2 \sigma^{2}}
$$

provided that $|w|<1$. See also A.G. Pakes [41].

Now let us define a sequence of random events $A_{0}, A_{1}, \ldots, A_{n}, \ldots$ in the following way: $A_{0}$ is the sure event, and $A_{n}(n=1,2, \ldots)$ occurs if and only if $N_{n}=n$. Then $\left\{A_{n}\right\}$ is a recurrent sequence of events in which $u_{0}=\mathbf{P}\left\{A_{0}\right\}=1$ and

$$
u_{n}=\mathbf{P}\left\{A_{n}\right\}=\mathbf{P}\left\{N_{n}=n\right\}
$$

for $n=1,2, \ldots$. Since $\operatorname{gcd}\left\{n: u_{n}>0\right\}=d$, the recurrent events are periodic with period $d$ if $d>1$. By the local central limit theorem of B.V. Gnedenko [18] we have

$$
u_{n} \sim d / \sqrt{2 \pi \sigma^{2} n}
$$

if $n=s d(s=1,2, \ldots)$ and $s \rightarrow \infty$.

Denote by $f_{n}(n=1,2, \ldots)$ the probability that the recurrence time is $n$. By (19), $f=f_{1}+f_{2}+\ldots+f_{n}+\ldots=1$ and thus the recurrent events are persistent. We note that $\operatorname{gcd}\left\{n: f_{n}>0\right\}=d$. If $|w|<1$, we have

$$
h(w)=\sum_{n=1}^{\infty} f_{n} w^{n}=w f^{\prime}(g(w))=1-\frac{g(w)}{w g^{\prime}(w)}
$$

and $h(1)=1$. This follows from the equations

$$
u_{n}=\sum_{i=1}^{n} f_{i} u_{n-i}
$$

and

$$
n \mathbf{P}\{\rho=n\}=\sum_{i=1}^{n} \mathbf{P}\{\rho=i\} u_{n-i}
$$

for $n \geq 1$. By (16) and (17) it follows that 


$$
h(w)=1-\sqrt{2 \sigma^{2}(1-w)}\left[1+\epsilon_{2}(w)\right]
$$

where $\epsilon_{2}(w) \rightarrow 0$ as $w \rightarrow 1(|w|<1)$. Hence by a Tauberian theorem

$$
f_{n}+f_{n+1}+\ldots \sim 2 \sigma / \sqrt{2 \pi n}
$$

as $n \rightarrow \infty$.

If we assume that $\theta_{1}, \theta_{2}, \ldots, \theta_{k}, \ldots$ are independent random variables such that

$$
\mathbf{P}\left\{\theta_{k}=n\right\}=f_{n}
$$

for $n \geq 1$ and $k \geq 1$, then by (24), the random variables $\theta_{1}, \theta_{2}, \ldots, \theta_{k}, \ldots$ belong to the domain of attraction of the stable distribution function

and

$$
G(x)=\left\{\begin{array}{cl}
2[1-\Phi(1 / \sqrt{x})] & \text { for } x>0, \\
0 & \text { for } x \leq 0,
\end{array}\right.
$$

$$
\lim _{k \rightarrow \infty} \mathbf{P}\left\{\frac{\theta_{1}+\theta_{2}+\ldots+\theta_{k}}{k^{2} \sigma^{2}} \leq x\right\}=G(x) .
$$

By the local limit theorem of B.V. Gnedenko [19] (B.V. Gnedenko and A.N. Kolmogorov [20] p. 236) it follows also that

$$
\lim _{k \rightarrow \infty}\left[k^{2} \sigma^{2} \mathbf{P}\left\{\theta_{1}+\theta_{2}+\ldots+\theta_{k}=n\right\}-d g\left(n / k^{2} \sigma^{2}\right)\right]=0
$$

uniformly with respect to $n=s d(s=1,2, \ldots)$. If $n$ is not divisible by $d$, then clearly $\mathbf{P}\left\{\theta_{1}+\ldots+\theta_{k}=n\right\}=0$. In (28)

$$
g(x)=G^{\prime}(x)=\frac{1}{\sqrt{2 \pi x^{3}}} e^{-1 / 2 x}
$$

for $x>0$. Accordingly,

$$
\mathbf{E}\left\{w^{\theta_{1}+\theta_{2}+\ldots+\theta_{k}}\right\}=[h(w)]^{k}
$$

for $|w| \leq 1$ and for every $\epsilon>0$ there exists a $K(\epsilon)$ such that

$$
\left|\mathbf{P}\left\{\theta_{1}+\ldots+\theta_{k}=n\right\}-d k \sigma e^{-k^{2} \sigma^{2} / 2 n} / \sqrt{2 \pi n^{3}}\right|<\epsilon / k^{2}
$$

for $k>K(\epsilon)$ and for every $n=s d(s=1,2, \ldots)$.

We note that if in (31) we replace $\theta_{1}$ by another discrete random variable whose possible values are integral multiples of $d$ and whose distribution is different from $\left\{f_{n}\right\}$, then (28) and (31) hold unchangeably. This immediately follows from the proof of the aforementioned theorem of B.V. Gnedenko [19]. 
Remarks: Throughout this paper we make the assumption (11), that is, $a=1$. However, the results proved in this paper are valid also for $a \neq 1$. Namely, if $a \neq 1$, the conditional distributions of the random variables $\xi(m), \mu$ and $\tau$, given that $\rho=n$, remain unchanged if we replace the probability distribution $\left\{p_{j}\right\}$ by $\left\{p_{j}^{*}\right\}$ where

$$
p_{j}^{*}=p_{j} c^{j} / f(c)
$$

and $c$ is any positive real number for which $f(c)<\infty$. Then

$$
a^{*}=\sum_{j=0}^{\infty} j p_{j}^{*}=c f^{\prime}(c) / f(c) .
$$

If there is a $c>0$ such that $a^{*}=1$, then in finding the conditional distributions of $\xi(m), \mu$ and $\tau$ given that $\rho=n$, we may substitute $\left\{p_{j}^{*}\right\}$ for $\left\{p_{j}\right\}$. If, in addition, $f^{\prime \prime}(c)<\infty$, then the variance of $\left\{p_{j}^{*}\right\}$ is also finite. If $a \leq 1$, then there always exists a $c$ such that $0 \leq c \leq 1$ and $a^{*}=1$.

\section{THE DISTRIBUTION OF $\xi(m)$}

It is assumed that (10), (11), (12) and (13) are satisfied. The conditional distribution of $\xi(m)$ given $\rho=n$ is determined by the generating function

$$
\Phi_{m}(z, w)=\mathbf{E}\left\{z^{\xi(m)} w^{\rho}\right\}
$$

defined for $|z| \leq 1$ and $|w| \leq 1$.

Theorem 1: If $|z| \leq 1$ and $|w| \leq 1$, we have

$$
\Phi_{m}(z, w)=w f\left(\Phi_{m-1}(z, w)\right)
$$

for $m=1,2, \ldots$ where $\Phi_{0}(z, w)=z g(w)$ and $g(w)$ is defined by (8).

Proof: Obviously,

$$
\Phi_{m}(z, w)=w \sum_{j=0}^{\infty} p_{j}\left[\Phi_{m-1}(z, w)\right]^{j}
$$

for $m=1,2, \ldots,|z| \leq 1$ and $|w| \leq 1$ where $\Phi_{0}(z, w)=z g(w)$. This implies (35). From (35) it follows that

$$
\frac{\partial \Phi_{m}(z, w)}{\partial z}=w f^{\prime}\left(\Phi_{m-1}(z, w)\right) \frac{\partial \Phi_{m-1}(z, w)}{\partial z}
$$

for $|z|<1$. If we let $z \rightarrow 1$, we obtain that

$$
\mathbf{E}\left\{\xi(m) w^{\rho}\right\}=w f^{\prime}(g(w)) \mathbf{E}\left\{\xi(m-1) w^{\rho}\right\}
$$


for $m=1,2, \ldots$. Hence by $(20)$ it follows that

$$
\mathbf{E}\left\{\xi(m) w^{\rho}\right\}=g(w)[h(w)]^{m}
$$

for $m=0,1,2, \ldots$ and $|w| \leq 1$. On the right-hand side of (39) both $g(w)$ and $h(w)$ are generating functions of discrete random variables taking on positive integers only. If we form the coefficient of $w^{n}$ in the Taylor expansion of (39) and divide it by $\mathbf{P}\{\rho=n\}$, then we can calculate $\mathbf{E}\{\xi(m) \mid \rho=n\}$ explicitly. The possible values of $\rho$ are $n=s d+1(s=0,1,2, \ldots)$. If $n=s d+1(s=0,1,2, \ldots)$ and if we use (15) and (31), we obtain from (39) that for any $\epsilon>0$

$$
\left|\mathrm{E}\{\xi(m) \mid \rho=n\}-m \sigma^{2} e^{-m^{2} \sigma^{2} / 2 n}\right|<\epsilon n^{3 / 2} / m^{2}
$$

for sufficiently large $m$ and $n$. If in (40), $m=[2 \alpha \sqrt{n} / \sigma]$ where $0<\alpha<\infty$, then we obtain that

$$
\lim _{n \rightarrow \infty} \mathrm{E}\left\{\frac{2 \xi([2 \alpha \sqrt{n} / \sigma])}{\sigma \sqrt{n}} \mid \rho=n\right\}=\mu_{1}(\alpha)
$$

exists and

$$
\mu_{1}(\alpha)=4 \alpha e^{-2 \alpha^{2}} \text {. }
$$

If we form the second derivative of (35) with respect to $z$ and let $z \rightarrow 1(|z|<1)$, then we obtain a recurrence formula for the determination of the second binomial moment of $\xi(m)$. Thus we obtain that

$$
\mathbf{E}\left\{\left(\begin{array}{c}
\xi(m) \\
2
\end{array}\right) w^{\rho}\right\}=\frac{w[g(w)]^{2} f^{\prime \prime}(g(w))}{2} \sum_{1 \leq i \leq m}[h(w)]^{m+i-2}
$$

for $m \geq 1$. If we form the coefficient of $w^{n}$ in the Taylor expansion of (43) and divide it by $\mathbf{P}\{\rho=n\}$, then we can calculate $\mathbf{E}\left\{\left(\begin{array}{c}\xi(m) \\ 2\end{array}\right) \mid \rho=n\right\}$ explicitly. If $n=s d+1(s=0,1,2, \ldots)$ and if we take into consideration that $[g(w)]^{2} f^{\prime \prime}(g(w)) / \sigma^{2}$ is the generating function of a discrete random variable whose possible values are $s d(s=1,2, \ldots)$, then by (15) and (31) we obtain that for any $\epsilon>0$

$$
\left|\mathbf{E}\left\{\left(\begin{array}{c}
\xi(m) \\
2
\end{array}\right) \mid \rho=n\right\}-\frac{\sigma^{4}}{2} \sum_{i=1}^{m}(m+i) e^{-(m+i)^{2} \sigma^{2} / 2 n}\right|<\epsilon n^{3 / 2} / m
$$

for sufficiently large $m$ and $n$. If in (44), $m=[2 \alpha \sqrt{n} / \sigma]$ where $0<\alpha<\infty$, then we obtain that

exists and

$$
\lim _{n \rightarrow \infty} \mathbf{E}\left\{\left(\frac{2 \xi([2 \alpha \sqrt{n} / \sigma])}{\sigma \sqrt{n}}\right)^{2} \mid \rho=n\right\}=\mu_{2}(\alpha)
$$

$$
\mu_{2}(\alpha)=16 \alpha^{2} \int_{0}^{1}(1+x) e^{-2 \alpha^{2}(1+x)^{2}} d x=4\left(e^{-2 \alpha^{2}}-e^{-8 \alpha^{2}}\right)
$$

We can continue the above procedure for $r=3,4, \ldots$ to obtain the following result. 
Theorem 2: If the following conditions are satisfied: $r \geq 0,0<\alpha<\infty$,

and $n=s d+1(s=0,1, \ldots)$, then

$$
\sum_{j=0}^{\infty} j^{r} p_{j}<\infty
$$

$$
\lim _{n \rightarrow \infty} \mathbf{E}\left\{\left(\frac{2 \xi([2 \alpha \sqrt{n} / \sigma])}{\sigma \sqrt{n}}\right)^{r} \mid \rho=n\right\}=\mu_{r}(\alpha)
$$

exists. We have $\mu_{0}(\alpha)=1, \mu_{1}(\alpha)=4 \alpha e^{-2 \alpha^{2}}$ and

for $r \geq 2$, where

$$
\mu_{r}(\alpha)=2^{r+1} r ! \alpha^{r} \int_{0}^{r-1}(1+x) e^{-2 \alpha^{2}(1+x)^{2}} g_{r-1}(x) d x
$$

$$
g_{r-1}(x)=\sum_{j=0}^{[x]}(-1)^{j}\left(\begin{array}{c}
r-1 \\
j
\end{array}\right) \frac{(x-j)^{r-2}}{(r-2) !}
$$

for $r \geq 2$ and $x \geq 0$.

Proof: Let us introduce the notation

$$
B_{r}^{m}(w)=\frac{1}{r !}\left(\frac{\partial^{r} \Phi_{m}(z, w)}{\partial z^{r}}\right)_{z=1}=\mathbf{E}\left\{\left(\begin{array}{c}
\xi(m) \\
r
\end{array}\right) w^{\rho}\right\}
$$

for $r \geq 0, m \geq 0$ and $|w| \leq 1$. We are interested in finding the asymptotic behavior of the coefficient of $w^{n}$ in the Taylor series of (51) in the case where $m=[2 \alpha \sqrt{n} / \sigma], 0<\alpha<\infty$, $n=s d+1(s=0,1,2, \ldots)$ and $n \rightarrow \infty$. We have already considered the cases where $r=1$ and $r=2$. Let us assume now that $r \geq 2$. If we form the $r$ th derivative of (35) with respect to $z$ and let $z \rightarrow 1(|z|<1)$, then we obtain

$$
\begin{gathered}
B_{r}^{m}(w)=h(w) B_{r}^{m-1}(w)+\frac{w f^{\prime \prime}(g(w))}{2} \sum_{1 \leq i \leq r} B_{i}^{m-1}(w) B_{r-i}^{m-1}(w)+\cdots \\
+\frac{w f^{(r)}(g(w))}{r !}\left[B_{1}^{m-1}(w)\right]^{r}
\end{gathered}
$$

Hence we obtain that

$$
\begin{gathered}
B_{r}^{m}(w)=\frac{w f^{\prime \prime}(g(w))}{2} \sum_{j=1}^{m} \sum_{1 \leq i<r}[h(w)]^{m-j_{B}} B_{i}^{j-1}(w) B_{r-i}^{j-1}(w)+\cdots \\
+\frac{w f^{(r)}(g(w))}{r !} \sum_{j=1}^{m}\left[B_{1}^{j-1}(w)\right]^{r}[h(w)]^{m-j}
\end{gathered}
$$

By this recurrence formula we can draw the conclusion that

$$
\begin{gathered}
B_{r}^{m}(w)=\frac{(r-1) ! w^{r-1}[g(w)]^{r}\left[f^{\prime \prime}(g(w))\right]^{r-1}}{2^{r-1}} \\
\sum_{1 \leq i_{1}<i_{2}<\ldots<i_{r-1} \leq m}[h(w)]^{m+i_{1}+\ldots+i_{r-1}-2(r-1)}+\ldots
\end{gathered}
$$


for $r \geq 2$ where each neglected term is the product of a constant, $w$, a generating function of a discrete random variable whose possible values are integral multiples of $d$, and a sum similar to the one displayed except that in these sums $i_{1}, i_{2}, \ldots, i_{r-1}$ are not distinct; for at least one $\nu=2, \ldots, r-1$ we have $i_{\nu}=i_{\nu-1}$. Consequently, the neglected terms in (54) do not contribute to the asymptotic expansion of the coefficient of $w^{n}$ in $B_{r}^{m}(w)$. The fact that the leading term in $B_{r}^{m}(w)$ is given by (54) can be proved by mathematical induction if we use (53). We note that (47) implies that $f^{(\nu)}(1)<\infty$ for $\nu \leq r$ and consequently $[g(w)]^{\nu} f^{(\nu)}(w) / f^{(\nu)}(1)$ is the generating function of a discrete random variable whose possible values are integral multiples of $d$.

By (15), (31) and (54) we can draw the conclusion that for every $\epsilon>0$

$$
\begin{gathered}
\mid \mathrm{E}\left\{\left(\begin{array}{c}
\xi(m) \\
r
\end{array}\right) \mid \rho=n\right\}-\frac{\sigma^{2 r}(r-1) !}{2^{r-1}} \\
\sum_{1 \leq i_{1}<i_{2}<\ldots<i_{r-1} \leq m}\left(m+i_{1}+\ldots+i_{r-1}\right) e^{-\left(m+i_{1}+\ldots+i_{r-1}\right)^{2} \sigma^{2} / 2 n} \mid<\epsilon n^{3 / 2} m^{r-3}
\end{gathered}
$$

if $r \geq 2$ and $m$ and $n$ are sufficiently large. If in (55) $m=[2 \alpha \sqrt{n} / \sigma]$ where $0<\alpha<\infty$, then we obtain that

$$
\lim _{n \rightarrow \infty} \mathrm{E}\left\{\left(\frac{2 \xi([2 \alpha \sqrt{n} / \sigma])}{\sigma \sqrt{n}}\right)^{r} \mid \rho=n\right\}=\mu_{r}(\alpha)
$$

exists for $r \geq 2$ and

$$
\begin{gathered}
\mu_{r}(\alpha)=(r-1) ! \alpha_{r} \\
\int_{0<x_{1}<\ldots<x_{r-1}<1}\left(1+x_{1}+\ldots+x_{r-1}\right) e^{-2 \alpha^{2}\left(1+x_{1}+\ldots+x_{r-1}\right)^{2}} d x_{1} \ldots d x_{r-1}
\end{gathered}
$$

or

$$
\mu_{r}(\alpha)=\alpha_{r} \int_{0}^{1} \ldots \int_{0}^{1}\left(1+x_{1}+\ldots+x_{r-1}\right) e^{-2 \alpha^{2}\left(1+x_{1}+\ldots+x_{r-1}\right)^{2}} d x_{1} \ldots d x_{r-1}
$$

where $\alpha_{r}=2^{r+1} r ! \alpha^{r}$. This proves (48) for $r \geq 2$. In (49) $g_{r-1}(x)$ is the density function of $\xi_{1}+\xi_{2}+\ldots+\xi_{r-1}$ where $\xi_{1}, \xi_{2}, \ldots, \xi_{r-1}$ are independent random variables each having a uniform distribution over the interval $(0,1)$. For the density function $g_{r-1}(x)$, formula (50) was found by P.S. Laplace [34], pp. 256-257. For a simple proof of (50) see L. Takács [48].

We note that

$$
\mu_{3}(\alpha)=12 \sqrt{2 \pi}[2 \Phi(4 \alpha)-\Phi(2 \alpha)-\Phi(6 \alpha)]
$$


and

$$
\begin{gathered}
\mu_{4}(\alpha)=48 \alpha\left[e^{-2 \alpha^{2}}-3 e^{-8 \alpha^{2}}+3 e^{-18 \alpha^{2}}-e^{-32 \alpha^{2}}\right] \\
+96 \sqrt{2 \pi} \alpha^{2}[\Phi(2 \alpha)-6 \Phi(4 \alpha)+9 \Phi(6 \alpha)-4 \Phi(8 \alpha)]
\end{gathered}
$$

where

$$
\Phi(x)=\frac{1}{\sqrt{2 \pi}} \int_{-\infty}^{x} e^{-u^{2} / 2} d u
$$

is the normal distribution function.

For the asymptotic distribution of $\xi(m)$ given $\rho=n$ we have the following result.

Theorem 3: $\quad$ If $0<\alpha<\infty$, if

$$
\sum_{j=0}^{\infty} j^{r} p_{j}<\infty
$$

for $r \geq 2$ and if $n=s d+1(s=0,1,2, \ldots)$, then

$$
\lim _{n \rightarrow \infty} \mathbf{P}\left\{\frac{2 \xi([2 \alpha \sqrt{n} / \sigma])}{\sigma \sqrt{n}} \leq x \mid \rho=n\right\}=G_{\alpha}(x)
$$

for $x>0$ where $G_{\alpha}(x)$ is the distribution function of a nonnegative random variable and is given by

$$
G_{\alpha}(x)=1-2 \sum_{j=1}^{\infty} \sum_{k=0}^{j-1}\left(\begin{array}{c}
j-1 \\
k
\end{array}\right) e^{-(x+2 \alpha j)^{2} / 2}(-x)^{k} H_{k+2}(x+2 \alpha j) / k !
$$

for $x \geq 0$ where $H_{0}(x), H_{1}(x) \ldots$ are the Hermite polynomials defined by

$$
H_{n}(x)=n ! \sum_{j=0}^{[n / 2]} \frac{(-1)^{j} x^{n-2 j}}{2^{j} j !(n-2 j) !} \text {. }
$$

We have

$$
G_{\alpha}(0)=1-2 \sum_{j=1}^{\infty}\left(4 \alpha^{2} j^{2}-1\right) e^{-2 \alpha^{2} j^{2}}
$$

and

$$
\frac{d G_{\alpha}(x)}{d x}=2 \sum_{j=1}^{\infty} \sum_{k=1}^{j}\left(\begin{array}{l}
j \\
k
\end{array}\right) e^{-(x+2 \alpha j)^{2} / 2}(-x)^{k-1} H_{k+2}(x+2 \alpha j) /(k-1) !
$$

if $x>0$.

Proof: $\quad$ Since

$$
u e^{-u^{2}} \leq(2 e)^{-1 / 2}<1 / 2
$$

if $u \geq 0$, it follows from (49) that

$$
\mu_{r}(\alpha) / r !<(2 \alpha)^{r} / \alpha
$$


for $r \geq 2$. Accordingly, there exists one and only one distribution function $G_{\alpha}(x)$ such that $G_{\alpha}(x)=0$ for $x<0$ and

$$
\int_{-0}^{\infty} x^{r} d G_{\alpha}(x)=\mu_{r}(\alpha)
$$

for $r \geq 0$. By the moment convergence theorem of M. Fréchet and J. Shohat [16] it follows from (48) that

$$
\lim _{n \rightarrow \infty} \mathbf{P}\left\{\frac{2 \xi([2 \alpha \sqrt{n} / \sigma])}{\sigma \sqrt{n}} \leq x \mid \rho=n\right\}=G_{\alpha}(x)
$$

in every continuity point of $G_{\alpha}(x)$. If $|s|<1 /(2 \alpha)$, then the Laplace-Stieltjes transform

$$
\Psi_{\alpha}(s)=\int_{-0}^{\infty} e^{-s x} d G_{\alpha}(x)
$$

can be expressed as

$$
\Psi_{\alpha}(s)=\sum_{r=0}^{\infty}(-1)^{r} \mu_{r}(\alpha) s^{r} / r !
$$

By (49) we obtain that

$$
\Psi_{\alpha}(s)=1+2 \sum_{k=1}^{\infty} \frac{(2 \alpha s)^{k}}{(k-1) !} \int_{k}^{\infty}\left(1-4 \alpha^{2} u^{2}\right)(u-k)^{k-1} e^{-2 \alpha^{2} u^{2}-2 \alpha(u-k) s} d u
$$

for $|s|<1 /(2 \alpha)$. Hence we obtain $(64)$ and (67) by inversion.

The limit distribution (63) has already been determined by D.P. Kennedy [26] in a different form. By his results we can conclude that

$$
\begin{aligned}
& G_{\alpha}(x)-G_{\alpha}(0)=\iint_{0<u<x /(2 \alpha)} e^{-\alpha^{2} u^{2} /\left(2\left(1-4 \alpha^{2} v\right)\right)}\left(1-4 \alpha^{2} v\right)^{-3 / 2} u f(u, v) d u d v \\
& 0<v<1 /\left(4 \alpha^{2}\right)
\end{aligned}
$$

for $x>0$ and

$$
\int_{0}^{\infty} \int_{0}^{\infty} e^{-s u-w v} f(u, v) d u d v=\left\{\frac{\sinh (\sqrt{2 w})}{\sqrt{2 w}}+s\left(\frac{\sinh (\sqrt{w / 2}}{\sqrt{w / 2}}\right)^{2}\right\}^{-1}
$$

for $\operatorname{Re}(s) \geq 0$ and $\operatorname{Re}(w) \geq 0$. See also A.G. Pakes [42].

We have $\mathbf{P}\{\mu<m \mid \rho=n\}=\mathbf{P}\{\xi(m)=0 \mid \rho=n\}$ and thus it follows from Theorem 3 that if $n=s d+1(s=0,1,2, \ldots)$, then

$$
\lim _{n \rightarrow \infty} \mathbf{P}\left\{\frac{\sigma \mu}{2 \sqrt{n}} \leq \alpha \mid \rho=n\right\}=G_{\alpha}(0)
$$

for $\alpha>0$ where $\mu$ is defined by (2) and $G_{\alpha}(0)$ is given by (66). For a direct proof of (77) we refer to V.F. Kolchin [31]. See also V.F. Kolchin [32] p. 128. 


\section{THE DISTRIBUTION OF $\tau$}

It is assumed that (10), (11), (12) and (13) are satisfied. The conditional distribution of $\tau$ given $\rho=n$ is determined by the generating function

$$
\Psi(z, w)=\mathbf{E}\left\{z^{\tau} w^{\rho}\right\}
$$

defined for $|z| \leq 1$ and $|w| \leq 1$.

Theorem 4: If $|z| \leq 1$ and $|w| \leq 1$, we have

$$
\Psi(z, w)=w f(\Psi(z, z w))
$$

Proof: Obviously,

$$
\mathbf{E}\left\{z^{\tau} w^{\rho}\right\}=w \sum_{j=0}^{\infty} p_{j}\left[\mathbf{E}\left\{z^{\tau+\rho} w^{\rho}\right\}\right]^{j}
$$

for $|z| \leq 1$ and $|w| \leq 1$. This implies (79).

In what follows we need the following auxiliary theorem.

Lemma 1: Let $q(w)$ be the generating function of a discrete random variable whose possible values are integral multiples of $d$ and let $h(w)$ be defined by (20). If $p=2,3, \ldots$ and

$$
\frac{w q(w)}{[1-h(w)]^{p}}=\sum_{n=0}^{\infty} c_{n} w^{n}
$$

for $|w|<1$, then

$$
c_{n} \sim \frac{d n^{(p-2) / 2}}{2^{p / 2} \Gamma(p / 2) \sigma^{p}}
$$

whenever $n=s d+1(s=0,1,2, \ldots)$ and $n \rightarrow \infty$.

Proof: $\quad$ Since $h(w) \neq 1$ if $w^{d} \neq 1$, and since by (23)

$$
\lim _{w \rightarrow 1}(1-w)^{p / 2} \frac{w q(w)}{[1-h(w)]^{p}}=\frac{1}{2^{p / 2} \sigma^{p}}
$$

(82) follows from a theorem of R. Jungen [24]. See also the Appendix.

If we form the derivative of (79) with respect to $z$ and let $z \rightarrow 1(|z|<1)$, we obtain that

$$
\mathbf{E}\left\{\tau w^{\rho}\right\}=w f^{\prime}(g(w)) \mathbf{E}\left\{(\tau+\rho) w^{\rho}\right\} .
$$

Hence we obtain that

$$
\mathbf{E}\left\{\tau w^{\rho}\right\}=\frac{w^{2} g^{\prime}(w) f^{\prime}(g(w))}{1-w f^{\prime}(g(w))}=\frac{g(w) h(w)}{[1-h(w)]^{2}}
$$


if $|w|<1 . \quad B y(16)$ and (23) it follows that

$$
\lim _{w \rightarrow 1}(1-w) \mathbf{E}\left\{\tau w^{\rho}\right\}=1 /\left(2 \sigma^{2}\right) .
$$

Hence by Lemma 1 if $n=s d+1(s=0,1,2, \ldots)$, then

$$
\lim _{n \rightarrow \infty} \mathbf{P}\{\rho=n\} \mathbf{E}\{\tau \mid \rho=n\}=d /\left(2 \sigma^{2}\right)
$$

and by (15)

$$
\lim _{n \rightarrow \infty} \frac{\sigma \mathbf{E}\{\tau \mid \rho=n\}}{2 n^{3 / 2}}=\sqrt{\frac{\pi}{8}}
$$

If we form the second derivative of $(79)$ with respect to $z$ and let $z \rightarrow 1(|z|<1)$ we obtain that

$$
\begin{gathered}
\mathbf{E}\left\{\tau^{2} w^{\rho}\right\}=\frac{g(w) h(w)\left[1+4 h(w)+h^{2}(w)\right]}{[1-h(w)]^{4}} \\
+\frac{w[g(w)]^{2} f^{\prime \prime}(g(w))\left[1+3 h(w)+h^{2}(w)\right]}{[1-h(w)]^{5}}
\end{gathered}
$$

for $|w|<1$. By (16) and (23) we get

$$
\begin{gathered}
\lim _{w \rightarrow 1}(1-w)^{5 / 2} \mathbf{E}\left\{\tau^{2} w^{\rho}\right\} \\
=\lim _{w \rightarrow 1} \frac{5 w(1-w)^{5 / 2}[g(w)]^{2} f^{\prime \prime}(g(w))}{[1-h(w)]^{5}}=\frac{5}{4 \sqrt{2} \sigma^{3}} .
\end{gathered}
$$

By Lemma 1 it follows from (90) that if $n=s d+1(s=0,1,2, \ldots)$, then

$$
\mathbf{E}\left\{\tau^{2} \mid \rho=n\right\} \mathbf{P}\{\rho=n\} \sim \frac{5 d n^{3 / 2}}{3 \sqrt{2 \pi} \sigma^{3}}
$$

and by (15)

$$
\lim _{n \rightarrow \infty} \frac{\sigma^{2} \mathbf{E}\left\{\tau^{2} \mid \rho=n\right\}}{4 n^{3}}=\frac{5}{12}
$$

We can continue the above procedure for $r=3,4, \ldots$ to obtain the following result.

\section{Theorem 5: If}

$$
\sum_{j=0}^{\infty} j^{r} p_{j}<\infty
$$

for $r \geq 2$, and $n=s d+1(s=0,1,2, \ldots)$, then the limit

exists for $r \geq 0$ and

$$
\begin{gathered}
\lim _{n \rightarrow \infty} \mathbf{E}\left\{\left(\frac{\sigma \tau}{2 n^{3 / 2}}\right)^{r} \mid \rho=n\right\}=M_{r} \\
M_{r}=K_{r} \frac{4 \sqrt{\pi} r !}{\Gamma((3 r-1) / 2) 2^{r / 2}}
\end{gathered}
$$


where $K_{0}=-1 / 2, K_{1}=1 / 8$ and

$$
K_{r}=\frac{3 r-4}{4} K_{r-1}+\sum_{j=1}^{r-1} K_{j} K_{r-j}
$$

for $r=2,3, \ldots$

Proof: By (88) and (92), the theorem is true for $r=1$ and $r=2$. Let us assume now that $r \geq 2$. By forming the $r$ th derivative of (80) with respect to $z$ and letting $z \rightarrow 1(|z|<1)$ we obtain that

$$
\begin{gathered}
\mathbf{E}\left\{\tau^{r} w^{\rho}\right\}=h(w) \mathbf{E}\left\{(\tau+\rho)^{r} w^{\rho}\right\} \\
+\frac{w f^{\prime \prime}(g(w))}{2} \sum_{i=1}^{r-1}\left(\begin{array}{c}
r \\
i
\end{array}\right) \mathbf{E}\left\{(\tau+\rho)^{i} w^{\rho}\right\} \mathbf{E}\left\{(\tau+\rho)^{r-i} w^{\rho}\right\}+\cdots \\
+w f^{(r)}(g(w))\left[\mathbf{E}\left\{(\tau+\rho) w^{\rho}\right\}\right]^{r}
\end{gathered}
$$

Hence

$$
\begin{aligned}
\mathbf{E}\left\{\tau^{r} w^{\rho}\right\}[1-h(w)] & =\frac{w f^{\prime \prime}(g(w))}{2} \sum_{i=1}^{r-1}\left(\begin{array}{c}
r \\
i
\end{array}\right) \mathbf{E}\left\{\tau^{i} w^{\rho}\right\} \mathbf{E}\left\{\tau^{r-i} w^{\rho}\right\} \\
& +r h(w) \mathbf{E}\left\{\tau^{r-1} \rho w^{\rho}\right\}+\cdots
\end{aligned}
$$

From (98) we can draw the conclusion that

$$
\mathbf{E}\left\{\tau^{r} w^{\rho}\right\}=\frac{K_{r} 2^{2 r+1} r ! w^{r-1}[g(w)]^{r}\left[f^{\prime \prime}(g(w))\right]^{r-1}}{[1-h(w)]^{3 r-1}}+\cdots
$$

where $K_{r}$ is a constant. If (93) is satisfied, then in (99) each neglected term has the form

$$
C w q(w)[1-h(w)]^{-p}
$$

where $C$ is a constant, $q(w)$ is the generating function of a discrete random variable whose possible values are integer multiples of $d$, and $p$ is an integer $<3 r-1$.

By (23) and (99) we obtain that

$$
\lim _{w \rightarrow 1}(1-w)^{(3 r-1) / 2} \mathbf{E}\left\{r^{r} w^{\rho}\right\}=K_{r} r 2^{(r+3) / 2} \sigma^{-(r+1)}
$$

for $r \geq 2$. By (86), (101) is true for $r=1$ if we define $K_{1}=1 / 8$. By (90) we obtain that $K_{2}=5 / 64$. If we multiply $(98)$ by $(1-w)^{3(r-1) / 2}$ and let $w \rightarrow 1$, we obtain $(96)$ for $r \geq 2$. By Lemma 1 we obtain from (101) that if $n=s d+1(s=0,1,2, \ldots)$, then

$$
\mathbf{E}\left\{\tau^{r} \mid \rho=n\right\} \mathbf{P}\{\rho=n\} \sim \frac{K_{r} r ! 2^{(r+3) / 2} d n^{3(r-1) / 2}}{\sigma^{r+1} \Gamma((3 r-1) / 2)}
$$

for $n \rightarrow \infty$, and by (15)

$$
\mathbf{E}\left\{\tau^{r} \mid \rho=n\right\} \sim \frac{\sqrt{2 \pi} K_{r} r ! 2^{(r+3) / 2}}{\Gamma((3 r-1) / 2) \sigma^{r}} n^{3 r / 2}
$$


for $n \rightarrow \infty$. This proves (94) for $r \geq 2$. If $r=1$, then (94) is true by (88). Since $M_{0}=1,(94)$ is true for $r=0$ too if we define $K_{0}=-1 / 2$. This completes the proof of the theorem.

For the asymptotic distribution of $\tau$ given $\rho=n$ we have the following result.

\section{Theorem 6: If}

$$
\sum_{j=0}^{\infty} j^{r} p_{j}<\infty
$$

for $r \geq 2$, and if $n=s d+1(s=0,1,2, \ldots)$, then

$$
\lim _{n \rightarrow \infty} \mathbf{P}\left\{\frac{\sigma \tau}{\sqrt{4 n^{3}}} \leq x \mid \rho=n\right\}=W(x)
$$

for $x \geq 0$ where $W(x)$ is the distribution function of a positive random variable and is given by

$$
W(x)=\frac{\sqrt{6}}{x} \sum_{k=1}^{\infty} e^{-v} v_{k}^{2 / 3} U\left(1 / 6,4 / 3, v_{k}\right)
$$

for $x>0, W(0)=0$ and

$$
W^{\prime}(x)=\frac{2 \sqrt{6}}{x^{2}} \sum_{k=1}^{\infty} e^{-v} k_{k}^{2 / 3} U\left(-5 / 6,4 / 3, v_{k}\right)
$$

for $x>0$ where $U(a, b, x)$ is the confluent hypergeometric function,

$$
v_{k}=2 a_{k}^{3} /\left(27 x^{2}\right)
$$

and $z=-a_{k}(k=1,2, \ldots)$ are the zeros of the Airy function Ai(z) arranged so that $0<a_{1}<a_{2}<\ldots<a_{k}<\ldots$

Proof: We can prove by (96) that

$$
\lim _{r \rightarrow \infty}\left(\frac{4}{3}\right)^{r} \frac{K_{r}}{(r-1) !}=\frac{1}{2 \pi}
$$

and this implies that

$$
M_{r} \sim \frac{6 r}{\sqrt{2}}\left(\frac{r}{12 e}\right)^{r / 2}
$$

as $r \rightarrow \infty$. Hence

$$
\sum_{r=1}^{\infty} M_{r}^{-1 / r}=\infty .
$$

By (94) the sequence $\left\{M_{r}\right\}$ is a moment sequence. Since the condition (111) is satisfied, we can conclude from a theorem of T. Carleman [4], [5] that there exists one and only one distribution function $W(x)$ such that $W(0)=0$ and

$$
\int_{0}^{\infty} x^{r} d W(x)=M_{r}
$$


holds for $r=0,1,2, \ldots$ By the moment convergence theorem of M. Fréchet and J. Shohat [16], (94) implies that (105) holds for every continuity point of $W(x)$.

Let

$$
\Psi(s)=\int_{0}^{\infty} e^{-s x} d W(x)
$$

be the Laplace-Stieltjes transform of $W(x)$. We have

$$
\Psi(s)=\sum_{r=0}^{\infty}(-1)^{r} M_{r} s^{r} / r !
$$

and the series is convergent on the whole complex plane. This follows from (110). If $s$ is a positive real number, then by (95) and (96) we can prove that

$$
\Psi(s)=\frac{s \sqrt{2 \pi}}{2 \pi i} \int_{C} \frac{A i^{\prime}(z)}{A i(z)} e^{z s^{2 / 3} / 2^{1 / 3}} d z
$$

where $C$ denotes integration along a contour which starts at infinity on the negative real $z$ axis, encircles the origin counter-clockwise, and returns its starting point. Here $A i(z)$ is the Airy function defined by

$$
\operatorname{Ai}(z)=\frac{1}{\pi} \int_{0}^{\infty} \cos \left(\frac{t^{3}}{3}+t z\right) d t .
$$

The function $A i(z)$ has zeros only on the negative real axis; namely $z=-a_{k}(k=1,2, \ldots)$ where $0<a_{1}<a_{2}<\ldots<a_{k}<\ldots$. By the theorem of residues, we obtain from (115) that

$$
\Psi(s)=s \sqrt{2 \pi} \sum_{k=1}^{\infty} e^{-a_{k} s^{2 / 3} / 2^{1 / 3}}
$$

for $s>0$. Hence we obtain (106) and (107) by inversion. For the definitions of the confluent hypergeometric function and the Airy function we refer to L.J. Slater [45], J.C.P. Miller [40] and M. Abramowitz and I.A. Stegun [1]. The first 50 zeros of $A i(z)$ and $A i^{\prime}(z)$ can be found in J.C.P. Miller [40], p. 43 for 8 decimals. See also M. Abramowitz and I.A. Stegun [1], p. 478.

\section{THE ALTITUDE OF RANDOM TREES}

The results derived for branching processes can also be used in the investigation of the distribution of the vertices of random rooted trees by altitude. A tree is a connected undirected graph which has no cycles, loops or multiple edges. A rooted tree has a vertex, the root, distinguished from the other vertices. The height of a vertex in a rooted tree is the distance from the vertex to the root, that is, the number of edges in the path from the vertex to the root. The total height of a rooted tree is the sum of the heights of its vertices. 
Let $S_{n}$ be a set of distinct rooted trees with $n$ vertices. Let us choose a tree at random in the set $S_{n}$, assuming that all the possible choices are equally probable. We shall consider different models of random rooted trees with $n$ vertices and define $\tau_{n}(m)$ as the number of vertices in layer $m$, that is, the number of vertices at a distance $m(m=0,1,2, \ldots)$ from the root and

$$
\tau_{n}=\sum_{m \geq 0} m \tau_{n}(m)
$$

as the total height of a tree chosen at random in $S_{n}$. The height of a random tree is

$$
\mu_{n}=\sup \left\{m: \tau_{n}(m)>0\right\}
$$

For several models of random trees we have

$$
\begin{gathered}
\mathbf{P}\left\{\tau_{n}(m)=k\right\}=\mathbf{P}\{\xi(m)=k \mid \rho=n\}, \\
\mathbf{P}\left\{\mu_{n}=k\right\}=\mathbf{P}\{\mu=k \mid \rho=n\}
\end{gathered}
$$

and

$$
\mathbf{P}\left\{\tau_{n}=k\right\}=\mathbf{P}\{\tau=k \mid \rho=n\}
$$

where $\{\xi(m), m \geq 0\}$ is a suitably chosen branching process and $\rho, \mu$ and $\tau$ are defined by (1), (2) and (3) respectively.

(i) Distinct rooted tress with n labeled vertices. In 1889, A. Cayley [7] observed that the number of distinct trees with $n$ labeled vertices is $n^{n-2}$. Since then various proofs have been found for Cayley's formula. For a simple proof see L. Takács [51]. The number of distinct rooted trees with $n$ labeled vertices is

$$
R_{n}=n^{n-1}
$$

for $n=1,2, \ldots$. Since among the $n$ vertices we can choose a root in $n$ ways, (123) immediately follows from Cayley's formula. Now denote by $S_{n}$ the set of distinct rooted trees with $n$ labeled vertices. In this case

$$
\lim _{n \rightarrow \infty} \mathbf{P}\left\{\frac{2 \tau_{n}([2 \alpha \sqrt{n}])}{\sqrt{n}} \leq x\right\}=G_{\alpha}(x)
$$

for $x>0$ and $0<\alpha<\infty$,

$$
\lim _{n \rightarrow \infty} \mathbf{P}\left\{\frac{\mu_{n}}{2 \sqrt{n}} \leq \alpha\right\}=G_{\alpha}(0)
$$

for $\alpha>0$ and

$$
\lim _{n \rightarrow \infty} \mathbf{P}\left\{\frac{\tau_{n}}{\sqrt{4 n^{3}}} \leq x\right\}=W(x)
$$


for $x \geq 0$.

If we consider a branching process $\{\xi(m), m \geq 0\}$ in which $\mathbf{P}\{\xi(0)=1\}=1$ and

$$
p_{j}=e^{-1} / j !
$$

for $j=0,1,2, \ldots$, then by $(120),(121),(122),(63),(77)$ and (105) we obtain (124), (125) and (126). Now $a=1, \sigma^{2}=1$ and $d=1$. For this model the limit distribution (124) has been found by V.E. Stepanov [46], the expectation of $\tau_{n}$ has been determined by J. Riordan and N.J.A. Sloane [44], and the limit distribution (125) by A. Rényi and G. Szekeres [43] and V.F. Kolchin [30].

(ii) Distinct rooted trees with $n+1$ unlabeled vertices. Denote by $C_{n}$ the number of distinct rooted (ordered) trees with $n+1$ unlabeled vertices. Obviously

$$
C_{n}=\sum_{i=1}^{n} C_{i-1} C_{n-i}
$$

for $n=1,2, \ldots$ where $C_{0}=1$. Hence it follows that

$$
C_{n}=\left(\begin{array}{c}
2 n \\
n
\end{array}\right) \frac{1}{n+1}
$$

the $n$th Catalan number. For other proofs of (129), see F. Harary, G. Prins and W. Tutte [21], N.G. de Bruijn and B.J.M. Morselt [13] and D.A. Klarner [27], [28]. Now denote by $S_{n+1}$ the set of distinct rooted (ordered) trees with $n+1$ unlabeled vertices. In this case

$$
\lim _{n \rightarrow \infty} \mathbf{P}\left\{\frac{2 \tau_{n+1}([\alpha \sqrt{2 n}])}{\sqrt{2 n}} \leq x\right\}=G_{\alpha}(x)
$$

for $x>0$ and $0<\alpha<\infty$,

$$
\lim _{n \rightarrow \infty} \mathbf{P}\left\{\frac{\mu_{n+1}}{\sqrt{2 n}} \leq \alpha\right\}=G_{\alpha}(0)
$$

for $\alpha>0$ and

$$
\lim _{n \rightarrow \infty} \mathbf{P}\left\{\frac{\tau_{n+1}}{\sqrt{2 n^{3}}} \leq x\right\}=W(x)
$$

for $x \geq 0$.

If we consider a branching process $\{\xi(m), m \geq 0\}$ in which $\mathbf{P}\{\xi(0)=1\}=1$ and

$$
p_{j}=1 / 2^{j+1}
$$

for $j=0,1,2, \ldots$, then by $(120),(121),(122),(63),(77)$ and (105) we obtain (130), (131) and (132). Now $a=1, \sigma^{2}=2$ and $d=1$. For this model the expectation of $\tau_{n+1}(m)$ has been determined by A. Meier and J.W. Moon [39], the expectation of $\tau_{n}$ by Ju. M. Voloshin [52], 
and the limit distribution (131) by I.V. Konovaltsev and E.P. Lipatov [33]. See also L. Takács [50]. N.G. de Bruijn, D.E. Knuth and S.O. Rice [12] demonstrated that

$$
\lim _{n \rightarrow \infty} \frac{\mathbf{E}\left\{\mu_{n+1}\right\}}{\sqrt{2 n}}=\sqrt{\frac{\pi}{2}}
$$

It is plausible that for $r>1$

where

$$
\lim _{n \rightarrow \infty} \mathbf{E}\left\{\left(\frac{\mu_{n+1}}{\sqrt{2 n}}\right)^{r}\right\}=\int_{0}^{\infty} \alpha^{r} d_{\alpha} G_{\alpha}(0)=\frac{2(r-1) \Gamma((r+2) / 2)}{2^{r / 2}} \zeta(r)
$$

$$
\zeta(r)=\sum_{n=1}^{\infty} \frac{1}{n^{r}}
$$

is the Riemann zeta function.

(iii) Distinct trivalent rooted trees with $2 n+1$ unlabeled vertices. In 1859, A. Cayley [6] proved that the number of different trivalent rooted trees with $2 n+1$ unlabeled vertices is the $n$th Catalan number $C_{n}$, defined by (129). In a trivalent rooted tree every vertex has degree 3 except the end vertices which have degree 1 and the root which has degree 2 . Now denote by $S_{2 n+1}$ the set of distinct trivalent rooted trees with $2 n+1$ unlabeled vertices $(n+1$ end vertices). In this case

$$
\lim _{n \rightarrow \infty} \mathbf{P}\left\{\frac{2 \tau_{2 n+1}([\alpha \sqrt{8 n}])}{\sqrt{2 n}} \leq x\right\}=G_{\alpha}(x)
$$

for $x>0$ and $0<\alpha<\infty$,

$$
\lim _{n \rightarrow \infty} \mathbf{P}\left\{\frac{\mu_{2 n+1}}{\sqrt{8 n}} \leq \alpha\right\}=G_{\alpha}(0)
$$

for $\alpha>0$ and

$$
\lim _{n \rightarrow \infty} \mathbf{P}\left\{\frac{\tau_{2 n+1}}{\sqrt{32 n^{3}}} \leq x\right\}=W(x)
$$

for $x \geq 0$.

If we consider a branching process $\{\xi(m), m \geq 0\}$ in which $\mathbf{P}\{\xi(0)=1\}=1$ and $p_{0}=p_{2}=1 / 2, \quad p_{j}=0$ for $j=1,3,4, \ldots$, then by (120), (121), (122), (63), (77) and (105) we obtain (137), (138), and (139). Now $a=1, \sigma^{2}=1$ and $d=2$.

(iv) Distinct rooted binary trees with $n$ vertices. A rooted binary tree with $n$ vertices is a tree imbedded in a full binary tree of depth $n$ in such a way that its root coincides with the root of the full binary tree. If $C_{n}$ denotes the number of distinct rooted binary trees with $n$ vertices, then $C_{n}$ satisfies (128) for $n \geq 1$ where $C_{0}=1$. Consequently, $C_{n}$ is the $n$th Catalan number defined by (129). Let us choose a tree at random among the $C_{n}$ distinct trees with $n$ vertices, assuming that all the possible choices are equally probable. Denote by $\tau_{n}^{*}(m)$, 
$\mu_{n}^{*}$ and $\tau_{n}^{*}$ respectively the number of vertices at a distance $m$ from the root, the height and the total height of a tree chosen at random. Then we have

$$
\lim _{n \rightarrow \infty} \mathbf{P}\left\{\frac{\sqrt{8} \tau_{n}^{*}([\alpha \sqrt{8 n}])}{\sqrt{n}} \leq x\right\}=G_{\alpha}(x)
$$

for $x>0$ and $0<\alpha<\infty$,

$$
\lim _{n \rightarrow \infty} \mathbf{P}\left\{\frac{\mu_{n}^{*}}{\sqrt{8 n}} \leq \alpha\right\}=G_{\alpha}(0)
$$

for $\alpha>0$ and

for $x \geq 0$.

$$
\lim _{n \rightarrow \infty} \mathbf{P}\left\{\frac{\tau_{n}^{*}}{\sqrt{8 n^{3}}} \leq x\right\}=W(x)
$$

There is a simple relation between binary trees and trivalent rooted trees. If $m \geq 0$, then $\tau_{n}^{*}(m)$ has the same distribution as $\tau_{2 n+1}(m) / 2$. If $n \geq 1$, then $\tau_{n}^{*}$ has the same distribution as $\left(\tau_{2 n+1}-2 n\right) / 2$, and if $n \geq 1$, then $\mu_{n}^{*}$ has the same distribution as $\mu_{2 n+1}-1$ where $\tau_{2 n+1}(m), \tau_{2 n+1}$ and $\mu_{2 n+1}$ are defined under (iii).

For this model the limit distribution (141) has been determined by $\mathrm{Ph}$. Flajolet and $\mathrm{A}$. Odlyzko [15] and G.G. Brown and B.O. Shubert [3].

\section{BROWNIAN EXCURSION}

Let $\left\{\eta^{+}(t), 0 \leq t \leq 1\right\}$ be a Brownian excursion process. Let

$$
\omega^{+}=\int_{0}^{1} \eta^{+}(t) d t
$$

and

$$
\gamma^{+}=\sup _{0 \leq t \leq 1}\left\{\eta^{+}(t)\right\}
$$

Furthermore, let $\tau^{+}(\alpha)$ be the local time at the level $\alpha$ for $\alpha \geq 0$. For the definition and properties of the Brownian excursion process we refer to P. Lévy [35], [36], K. Itô and H.P. McKean, Jr. [23], and K.L. Chung [8].

Now we shall prove that

$$
\mathbf{P}\left\{\tau^{+}(\alpha) \leq x\right\}=G_{\alpha}(x)
$$

if $x>0$ and $\alpha>0$,

$$
\mathbf{P}\left\{\gamma^{+} \leq \alpha\right\}=G_{\alpha}(0)
$$

for $\alpha>0$, and 


$$
\mathbf{P}\left\{\omega^{+} \leq x\right\}=W(x)
$$

for $x \geq 0$. In addition,

$$
\mathbf{E}\left\{\left[\tau^{+}(\alpha)\right]^{r}\right\}=\mu_{r}(\alpha)
$$

for $r \geq 0$ where $\mu_{r}(\alpha)$ is defined in Theorem 2 and

$$
\mathbf{E}\left\{\left(\omega^{+}\right)^{r}\right\}=M_{r}
$$

for $r \geq 0$ where $M_{r}$ is defined in Theorem 5 .

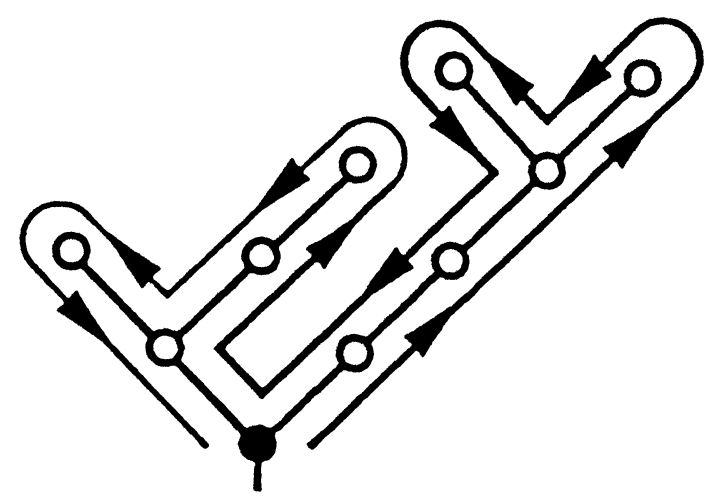

Figure 1: Traversing a tree

All these results can be deduced from the results of $(i i)$ for rooted trees with $n+1$ unlabeled vertices. Let us suppose that in the set $S_{n+1}$ the edges of the trees have unit lengths. Let us choose a tree at random in $S_{n+1}$ and suppose that a bug, starting at the root of the tree, crawls all over the branches of the tree. See Figure 1. Suppose that the bug crawls with unit velocity and follows the edges of the tree, always keeping to the right, until it returns to the root again. In this way the bug traverses all the $n$ edges twice. Denote by $\eta_{n}^{+}(t)$ the distance of the bug from the root at time $2 n t$ where $0 \leq t \leq 1$. The distance is measured along the edges of the tree. The process $\left\{\eta_{n}^{+}(t), 0 \leq t \leq 1\right\}$ is a Bernoulli excursion and $\left\{\eta_{n}^{+}(t) / \sqrt{2 n}, 0 \leq t \leq 1\right\}$ converges weakly to the Brownian excursion as $n \rightarrow \infty$.

Since

$$
\tau_{n+1}=\frac{n}{2}+n \int_{0}^{1} \eta_{n}^{+}(t) d t
$$


and since the integral is a continuous functional on the process it follows from (150) that

$$
\lim _{n \rightarrow \infty} \mathbf{P}\left\{\frac{\tau_{n+1}}{\sqrt{2 n^{3}}} \leq x\right\}=\mathbf{P}\left\{\omega^{+} \leq x\right\} .
$$

By (132) this proves (147). In a similar way we obtain that

$$
\lim _{n \rightarrow \infty} \mathbf{P}\left\{\frac{2 \tau_{n+1}([\alpha \sqrt{2 n}])}{\sqrt{2 n}} \leq x\right\}=\mathbf{P}\left\{\tau^{+}(\alpha) \leq x\right\} .
$$

for $0<\alpha<\infty$. By (130) this proves (145). Furthermore

$$
\lim _{n \rightarrow \infty} \mathbf{P}\left\{\frac{\mu_{n+1}}{\sqrt{2 n}} \leq \alpha\right\}=\mathbf{P}\left\{\gamma^{+} \leq \alpha\right\}
$$

for $\alpha>0$. By (131) this proves (146). Formulas (148) and (149) follow from (48) and (94) respectively.

The distribution function of $\tau^{+}(\alpha)$ has been studied extensively. It has been expressed in the form of a complex integral by R.K. Getoor and M.J. Sharpe [17], J.W. Cohen and G. Hooghiemstra [9], G. Louchard [37], E. Csáki and S.G. Mohanty [10], and Ph. Biane and M. Yor [2]. F.B. Knight [29] and G. Hooghiemstra [22] expressed $\mathbf{P}\left\{\tau^{+}(\alpha) \leq x\right\}$ in explicit forms, but their formulas are too complicated for numerical calculations. The Laplace transform of $\mathbf{P}\left\{\omega^{+} \leq x\right\}$ has been determined by D.A. Darling [11] and G. Louchard [38], and by numerical integration G. Louchard [38] calculated $\mathrm{P}\left\{\omega^{+} \leq x\right\}$ for $0.25<x<1.35$. By using formulas (64), (67), (106) and (107) and the remarkable program MATHEMATICA by S. Wolfram [53], we can easily produce tables and graphs for $G_{\alpha}(x), G_{\alpha}^{\prime}(x), W(x)$ and $W^{\prime}(x)$.

\section{APPENDIX}

In what follows, we shall give detailed proofs for formulas (16), (17), (23), (24) and (82). We can use the following Abelian theorem to prove (16) and (17).

Theorem 7: $\quad$ Let

$$
a(z)=\sum_{n=0}^{\infty} a_{n} z^{n}
$$

where $a_{n} \geq 0$,

$$
a_{n} \sim A \frac{n^{\alpha-1}}{\Gamma(\alpha)} \sim A\left(\begin{array}{c}
n+\alpha-1 \\
n
\end{array}\right)=A(-1)^{n}\left(\begin{array}{c}
n \\
n
\end{array}\right)
$$

as $n \rightarrow \infty$ where $A>0$ and $\alpha>0$. Then

$$
a(z) \sim A(1-z)^{-\alpha}
$$

as $z \rightarrow 1(|z|<1)$.

For the proof of this theorem we refer to W. Feller [A1] p. 423. 
By (15) we have

$$
\mathbf{P}\{\rho>n\} \sim \frac{2}{\sqrt{2 \pi \sigma^{2} n}}
$$

as $n \rightarrow \infty$ and thus by Theorem 7

$$
\sum_{n=0}^{\infty} \mathbf{P}\{\rho>n\} w^{n}=\frac{1-g(w)}{1-w} \sim \frac{2}{\sqrt{2 \sigma^{2}(1-w)}}
$$

as $w \rightarrow 1(|w|<1)$. This proves (16).

By (15) we have also

$$
\mathbf{P}\left\{N_{n}=n-1\right\} \sim \frac{d}{\sqrt{2 \pi \sigma^{2} n}} \sim \frac{d(-1)^{s}}{\sqrt{2 \sigma^{2} d}}\left(\begin{array}{c}
-1 / 2 \\
s
\end{array}\right)
$$

if $n=s d+1(s=0,1,2, \ldots)$ and $s \rightarrow \infty$. Thus by Theorem 7

$$
\sum_{n=1}^{\infty} \mathrm{P}\left\{N_{n}=n-1\right\} w^{n-1}=g^{\prime}(w) \sim \frac{d}{\sqrt{2 \sigma^{2} d\left(1-w^{d}\right)}} \sim \frac{1}{\sqrt{2 \sigma^{2}(1-w)}}
$$

as $w \rightarrow 1(|w|<1)$. Clearly $\left(1-w^{d}\right) \sim d(1-w)$ as $w \rightarrow 1$. This proves (17).

Formula (23) can also be proved by Theorem 7. By (19)

$$
u_{n} \sim \frac{d}{\sqrt{2 \pi \sigma^{2} n}} \sim \frac{d(-1)^{s}}{\sqrt{2 \sigma^{2} d}}\left(\begin{array}{c}
-1 / 2 \\
s
\end{array}\right)
$$

if $n=s d(s=0,1,2, \ldots)$ and $s \rightarrow \infty$. Consequently,

$$
\sum_{n=0}^{\infty} u_{n} w^{n}=\frac{1}{1-h(w)} \sim \frac{d}{\sqrt{2 \sigma^{2} d\left(1-w^{d}\right)}} \sim \frac{1}{\sqrt{2 \sigma^{2}(1-w)}}
$$

as $w \rightarrow 1(|w|<1)$. This proves (23).

We can use the following Tauberian theorem to prove (24).

Theorem 8: $\quad$ If $a_{0}, a_{1}, \ldots, a_{n}, \ldots$ are nonnegative real numbers, if

$$
a(z)=\sum_{n=0}^{\infty} a_{n} z^{n}
$$

is convergent for $|z|<1$ and if

$$
\lim _{z \rightarrow 1}(1-z)^{\alpha} a(z)=A
$$

where $\alpha>0$, then

$$
\lim _{n \rightarrow \infty} \frac{a_{0}+a_{1}+\cdots+a_{n}}{n^{\alpha}}=\frac{A}{\Gamma(\alpha+1)} .
$$


For the proof of this theorem, we refer to W. Feller [A1] p. 243.

Let $a_{n}=f_{n+1}+f_{n+2}+\ldots$ for $n \geq 0$. Then by (23)

$$
\sum_{n=0}^{\infty} a_{n} w^{n}=\frac{1-h(w)}{1-w} \sim \sqrt{\frac{2 \sigma^{2}}{1-w}}
$$

if $w \rightarrow 1(|w|)<1)$. Hence by Theorem 8

$$
\lim _{n \rightarrow \infty} \frac{a_{0}+a_{1}+\ldots+a_{n}}{\sqrt{n}}=\frac{2 \sqrt{2 \sigma^{2}}}{\sqrt{\pi}} .
$$

Since $a_{n} \leq a_{n-1}$ for $n \geq 1$, by (167) it follows that

$$
\lim _{n \rightarrow \infty} \sqrt{n} a_{n}=\sqrt{2 \sigma^{2} / \pi}
$$

This proves (24).

Formula (82) can be proved directly by using the following two auxiliary theorems.

Lemma 2: Let

$$
\gamma_{n}=\sum_{i=0}^{n} \alpha_{i} \beta_{n-i}
$$

for $n=0,1,2, \ldots$ where $\alpha_{n}>0$ and $\beta_{n}>0$. Let us suppose that $\alpha_{n} / \gamma_{n} \rightarrow 0, \beta_{n} / \gamma_{n} \rightarrow 0$ and $\gamma_{n-1} / \gamma_{n} \rightarrow 1$ as $n \rightarrow \infty$. If $a_{n} \sim \alpha_{n}$ and $b_{n} \sim \beta_{n}$ as $n \rightarrow \infty$, then

$$
\sum_{i=0}^{n} a_{i} b_{n-i} \sim \sum_{i=0}^{n} \alpha_{i} \beta_{n-i}
$$

as $n \rightarrow \infty$.

Proof: For any $\epsilon>0$ there exists a positive integer $m=m(\epsilon)$ such that $\left|a_{n}-\alpha_{n}\right|<\epsilon \alpha_{n}$ and $\left|b_{n}-\beta_{n}\right|<\epsilon \beta_{n}$ if $n>m$. Also there exists a constant $C$ such that $\left|a_{n}-\alpha_{n}\right|<C \alpha_{n}$ and $\left|b_{n}-\beta_{n}\right|<C \beta_{n}$ for all $n \geq 0$.

If $0 \leq i \leq n$, then

$$
\begin{gathered}
a_{i} b_{n-i}-\alpha_{i} \beta_{n-i}=\left(a_{i}-\alpha_{i}\right)\left(b_{n-i}-\beta_{n-i}\right) \\
+\left(a_{i}-\alpha_{i}\right) \beta_{n-i}+\alpha_{i}\left(b_{n-i}-\beta_{n-i}\right) .
\end{gathered}
$$

If $m>2 m$ and if we use the aforementioned inequalities in (171), we obtain that

$$
\begin{gathered}
\left|\sum_{i=0}^{n} a_{i} b_{n-i}-\sum_{i=0}^{n} \alpha_{i} \beta_{n-i}\right| \leq \epsilon(C+2) \gamma_{n}+ \\
C \sum_{i=0}^{m}\left(\alpha_{i} \beta_{n-i}+\alpha_{n-i} \beta_{i}\right) .
\end{gathered}
$$


By assumption

$$
\lim _{n \rightarrow \infty} \frac{\alpha_{i} \beta_{n-i}+\alpha_{n-i} \beta_{i}}{\gamma_{n}}=0
$$

for $0 \leq i \leq m$. Consequently, by (172)

$$
\lim _{n \rightarrow \infty} \sup \left|\frac{1}{\gamma_{n}} \sum_{i=0}^{n} a_{i} b_{n-i}-1\right|<\epsilon(C+2)
$$

for any $\epsilon>0$. This proves (170).

In a more general setting Lemma 2 has been proved by K. Knopp [A2].

Lemma 3: Let us suppose that $a_{0}, a_{1}, \ldots, a_{n}, \ldots$ are nonnegative real numbers with sum

$$
\sum_{n=0}^{\infty} a_{n}=1
$$

and that $b_{0}, b_{1}, \ldots, b_{n}, \ldots$ are real numbers for which

$$
\lim _{n \rightarrow \infty} b_{n} n^{-\beta}=b
$$

where $\beta \geq 0$ and $b>0$. If

$$
c_{n}=\sum_{i=0}^{n} a_{i} b_{n-i}
$$

for $n=0,1,2, \ldots$, then

$$
\lim _{n \rightarrow \infty} c_{n} n^{-\beta}=b
$$

Proof: Let $0<\epsilon<b / 2$ and choose an $m=m(\epsilon)$ so large that

$$
\sum_{k>m} a_{k}<\epsilon
$$

and

$$
\left|b_{n} n-\beta\right|<\epsilon
$$

if $n>m$. If $n>2 m$, then

Hence if $\beta \geq 0$, then

$$
\begin{gathered}
\frac{c_{n}}{b_{n}}-1=\sum_{k \leq m} a_{k}\left(\frac{b_{n-k}}{b_{n}}-1\right)+\sum_{m<k<n-m} a_{k} \frac{b_{n-k}}{b_{n}} \\
\quad+\sum_{n-m \leq k \leq n} a_{k} \frac{b_{n-k}}{b_{n}}-\sum_{k>m} a_{k} .
\end{gathered}
$$

$$
\lim _{n \rightarrow \infty} \sup \left|\frac{c_{n}}{b_{n}}-1\right| \leq 5 \epsilon / 2
$$

for $0<\epsilon<b / 2$. On the right-hand side of (181) in the first sum $b_{n-k} / b_{n} \rightarrow 1$ if $k \leq m$ and $n \rightarrow \infty$. In the second sum 


$$
\left|\frac{b_{n-k}}{b_{n}}\right|<\frac{(b+\epsilon)(n-k)^{\beta}}{(b-\epsilon) n^{\beta}} \leq \frac{b+\epsilon}{b-\epsilon} \leq \frac{3}{2}
$$

if $m<k<n-m$. The third sum

$$
\sum_{n-m \leq k \leq n} a_{k} \frac{b_{n-k}}{b_{n}}=\frac{1}{b_{n_{i}}} \sum_{=0}^{m} b_{i} a_{n-i} \rightarrow 0
$$

as $n \rightarrow \infty$. The fourth sum has absolute value $<\epsilon$. Hence (182) and (178) follow.

Another version of Lemma 3 has been proved by O. Szász [A3].

If in Lemma 2

$$
\alpha_{n}=\left(\begin{array}{c}
\alpha+n-1 \\
n
\end{array}\right) \sim \frac{n^{\alpha-1}}{\Gamma(\alpha)}
$$

and

$$
\beta_{n}=\left(\begin{array}{c}
\beta+n-1 \\
n
\end{array}\right) \sim \frac{n^{\beta-1}}{\Gamma(\beta)}
$$

where $\alpha>0$ and $\beta>0$, then

$$
\gamma_{n}=\left(\begin{array}{c}
\alpha+\beta+n-1 \\
n
\end{array}\right) \sim \frac{n^{\alpha+\beta-1}}{\Gamma(\alpha+\beta)}
$$

Accordingly, if in (177)

$$
a_{n} \sim n^{\alpha-1} / \Gamma(\alpha)
$$

as $n \rightarrow \infty$ where $\alpha>0$, and if

$$
b_{n} \sim n^{\beta-1} / \Gamma(\beta)
$$

as $n \rightarrow \infty$ where $\beta>0$, then

$$
c_{n} \sim n^{\alpha+\beta-1} / \Gamma(\alpha+\beta)
$$

as $n \rightarrow \infty$.

Furthermore, it follows from the above formulas that

for $p=1,2, \ldots$ as $n \rightarrow \infty$.

$$
\sum_{i_{i}+\ldots+i_{p}=n} a_{i_{1}} a_{i_{2}} \ldots a_{i_{p}} \sim n^{\alpha p-1} / \Gamma(\alpha p)
$$

If in (191)

$$
a_{n}=\sqrt{2 \sigma^{2}} u_{n d} / d
$$

for $n=0,1,2, \ldots$ where $u_{n}$ is defined by (18), then (188) is satisfied with $\alpha=1 / 2$ and (191) proves $(82)$ in the particular case where $q(w)=1$ and $p \geq 1$. If $p \geq 2$ and $q(w)$ is defined in Lemma 1 , then $(82)$ is true by Lemma 3. 
[A1] W. Feller, "An Introduction to Probability Theory and Its Applications", Vol. II, John Wiley and Sons, New York, 1966.

[A2] K. Knopp, “Über Summen der Form $a_{0} b_{n}+a_{1} b_{n-1}+\ldots+a_{n} b_{0}$ ", Rendiconti del Circolo Matematico di Palermo 32, (1911), pp. 95-110.

[A3] O. Szász, “Ein Grenzwertsatz über Potenzreihen”, Sitzungsberichte der Berliner Mathematischen Gesellschaft 21, (1921), pp. 25-29.

\section{REFERENCES}

[1] M. Abramowitz and I.A. Stegun, "Handbook of Mathematical Functions", Dover, New York, 1965.

[2] Ph. Biane and M. Yor, "Valeurs principales associées aux temps locaux browniens", Bulletin des Sciences Mathématiques 111, (1987), pp. 23-101.

[3] G.G. Brown and B.O. Shubert, "On random binary trees", Mathematics of Operations Research 9, (1984), pp. 43-65.

[4] T. Carleman, "Sur le problème des moments", Comptes Rendus Acad. Sci. Paris 174, (1922), pp. 1680-1682.

[5] T. Carleman, “Les Fonctions Quasi-Analytiques”, Gauthier-Villars, Paris, 1926.

[6] A. Cayley, "On the analytic forms called trees, Second part", Philosophical Magazine 18, (1859), pp. 374-378. [The Collected Mathematical Papers of Arthur Cayley, Vol. IV, Cambridge University Press, 1891, pp. 112-115].

[7] A. Cayley, "A theorem on trees", Quarterly Journal of Pure and Applied Mathematics 23, (1889), pp. 376-378. [The Collected Mathematical Papers of Arthur Cayley, Vol. XIII, Cambridge University Press, 1897, pp. 26-28].

[8] K.L. Chung, "Excursions in Brownian motion", Arkiv för Matematik 14, (1976), pp. 157-179.

[9] J.W. Cohen and G. Hooghiemstra, "Brownian excursion, the $M / M / 1$ queue and their occupation times", Mathematics of Operations Research 6, (1981), pp. 608-629.

[10] E. Csáki and S.G. Mohanty, "Some joint distributions for conditional random walks", The Canadian Journal of Statistics 14, (1986), pp. 19-28.

[11] D.A. Darling, "On the supremum of a certain Gaussian process", The Annals of Probability 11, (1983), pp. 803-806.

[12] N.G. de Bruijn, D.E. Knuth and S.O. Rice, "The average height of planted plane trees", Graph Theory and Computing, edited by R.C. Read, Academic Press, New York, 1972, pp. 15-22.

[13] N.G. de Bruijn and B.J.M. Morselt, "A note on plane trees", Journal of Combinatorial Theory 2, (1967), pp. 27-34. 
[14] M. Dwass, "The total progeny in a branching process and a related random walk", Journal of Applied Probability 6, (1969), pp. 682-686.

[15] Ph. Flajolet and A. Odlyzko, "The average height of binary trees and other simple trees", Journal of Computer and System Sciences 25, (1982), pp. 171-213.

[16] M. Fréchet and J. Shohat, "A proof of the generalized second-limit theorem in the theory of probability", Transactions of the American Mathematical Society 33, (1931), pp. 533-543.

[17] R.K. Getoor and M.J. Sharpe, "Excursions of Brownian motion and Bessel processes", Zeitschrift für Wahrscheinlichkeitstheorie und verwandte Gebiete 47, (1979), pp. 83-106.

[18] B.V. Gnedenko, "On the local limit theorem in probability theory", (Russian), Uspekhi Matematicheskih Nauk 3, No. 3, (1948), pp. 187-194.

[19] B.V. Gnedenko, "On the local limit theorem for stable limit distributions", (Russian), Ukranskii Matematicheskii Zhurnal No. 4, (1949), pp. 3-15.

[20] B.V. Gnedenko and A.N. Kolmogorov, "Limit Distributions for Sums of Independent Random Variables, Addison-Wesley, Cambridge, Mass., 1954.

[21] F. Harary, G. Prins, and W.T. Tutte, "The number of plane trees", Nederlandse Akademie van Wetenschappen, Proceedings, Ser. A, 67, (1964), pp. 319-329.

[22] G. Hooghiemstra, "On the explicit form of the density of Brownian excursion local time”, Proceedings of the American Mathematical Society, 84, (1982), pp. 127-130.

[23] K. Itô and H.P. McKean, Jr., "Diffusion Processes and their Sample Paths", SpringerVerlag, Berlin, 1965.

[24] R. Jungen, "Sur les séries de Taylor n'ayant que des singularités algébricologarithmiques sur leur cercle de convergence”, Commentarii Mathematica Helvetici 3, (1931), pp. 266-306.

[25] D.G. Kendall, "Some problems in the theory of queues", Journal of the Royal Statistical Society, Ser. B, 13, (1951), pp. 151-185.

[26] D.P. Kennedy, "The Galton-Watson process conditioned on total progeny", Journal of Applied Probability, 12, (1975), pp. 800-806.

[27] D.A. Klarner, “A correspondence between two sets of trees", Nederlandse Akademie Wetenschappen, Proceedings, Ser. A, 72, (1969), pp. 292-296.

[28] D.A. Klarner, "Correspondences between plane trees and binary sequences", Journal of Combinatorial Theory, 9, (1970), pp. 401-411.

[29] F.B. Knight, "On the excursion process of Brownian motion", Transactions of the American Mathematical Society, 258, (1980), pp. 77-86. [Correction: Zentralblatt für Mathematik 426, (1980), \#60073]. 
[30] V.F. Kolchin, "Branching processes, random trees, and a generalized scheme of arrangements of particles", Mathematical Notes 2, (1977), pp. 386-394.

[31] V.F. Kolchin, "Moment of degeneration of a branching process and height of a random tree", Mathematical Notes 24, (1978), pp. 954-961.

[32] V.F. Kolchin, “Random Mappings”, Optimization Software, Inc., New York, 1986.

[33] I.V. Konovaltsev and E.P. Lipatov, "Some properties of plane rooted trees", Cybernetics 6, (1970), pp. 660-667.

[34] P.S. Laplace, “Théorie analytique des probabilités", Paris, 1812. [Oeuvres VII, Gauthier-Villars, Paris, 1886, Culture et Civilisation, Bruxelles, 1967].

[35] P. Lévy, "Sur certains processus stochastiques homogènes", Compositio Mathematica 7, (1940), pp. 283-339.

[36] P. Lévy, "Processus Stochastiques et Mouvement Brownien", Second edition, GauthierVillars, Paris, 1965.

[37] G. Louchard, “Kac's formula, Lévy's local time and Brownian excursion”, Journal of Applied Probability 21, (1984), pp. 479-499.

[38] G. Louchard, "The Brownian excursion area: a numerical analysis", Computers and Mathematics with Applications 10, (1984), pp. 413-417. [Erratum: Ibid A 12 (1986), $375]$.

[39] A. Meir and J.W. Moon, "On the altitude of nodes in random trees", Canadian Journal of Mathematics 30, (1978), pp. 997-1015.

[40] J.C.P. Miller, "The Airy Integral”, Cambridge University Press, 1946.

[41] A.G. Pakes, "Some limit theorems for the total progeny of a branching process", Journal of Applied Probability 3, (1971), pp. 176-192.

[42] A.G. Pakes, "Further results on the critical Galton-Watson process with immigration", Journal of the Australian Mathematical Society 13, (1972), pp. 277-290.

[43] A. Rényi and G. Szekeres, "On the height of trees", The Journal of the Australian Mathematical Society 7, (1967), pp. 497-507.

[44] J. Riordan and N.J.A. Sloane, "The enumeration of rooted trees by total height", Journal of the Australian Mathematical Society 10, (1969), pp. 278-282.

[45] L.J. Slater, “Confluent Hypergeometric Functions", Cambridge University Press, 1950.

[46] V.E. Stepanov, "On the distribution of the number of vertices in strata of a random tree", Theory of Probability and its Applications 14, (1969), pp. 65-78.

[47] L. Takács, "A generalization of the ballot problem and its application in the theory of queues, Journal of the American Statistical Association 57, (1962), pp. 327-337. 
[48] L. Takács, "On the method of inclusion and exclusion", Journal of the American Statistical Association 62, (1967), pp. 102-113.

[49] L. Takács, "Ballots, queues, and random graphs", Journal of Applied Probability 26, (1989), pp. 103-112.

[50] L. Takács, "Queues, random graphs, and branching processes", Journal of Applied Mathematics and Simulation 1, (1988), pp. 223-243.

[51] L. Takács, “On Cayley's formula for counting forests”, Journal of Combinatorial Theory, Ser. A, 53, (1990), pp. 321-323.

[52] Ju.M. Voloshin, "Enumeration of the terms of the object domains according to the depth of embedding", Soviet Mathematics-Doklady 15, (1974), pp. 1777-1782.

[53] S. Wolfram, "Mathematica. A System for Doing Mathematics by Computer", AddisonWesley, Redwood City, California, 1988. 


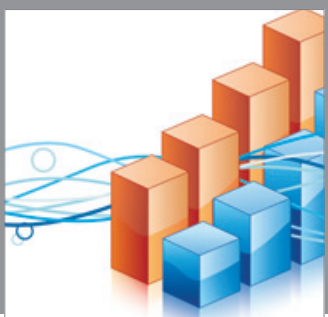

Advances in

Operations Research

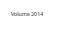

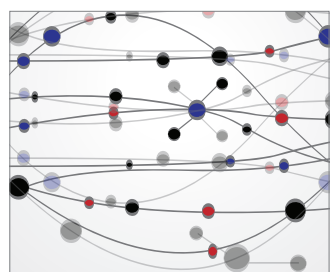

\section{The Scientific} World Journal
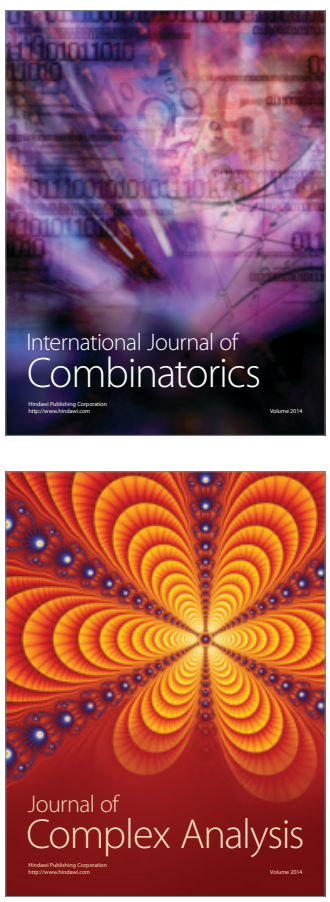

International Journal of

Mathematics and

Mathematical

Sciences
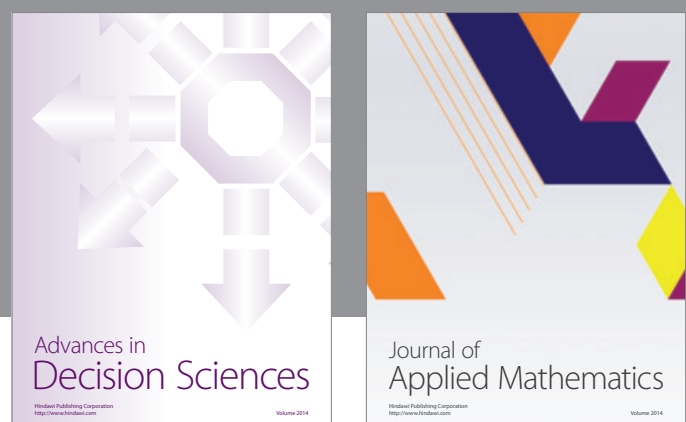

Journal of

Applied Mathematics
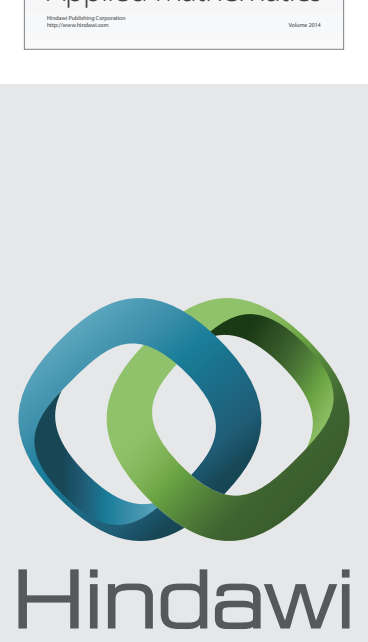

Submit your manuscripts at http://www.hindawi.com
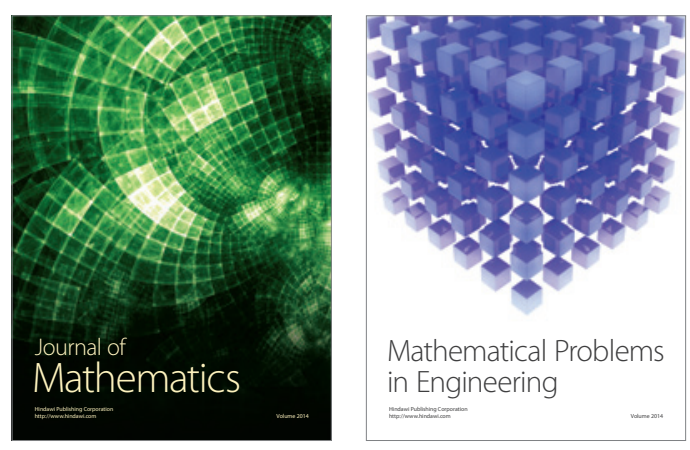

Mathematical Problems in Engineering
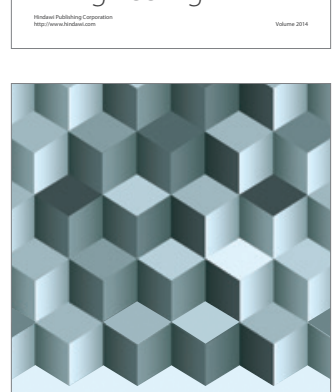

Journal of

Function Spaces
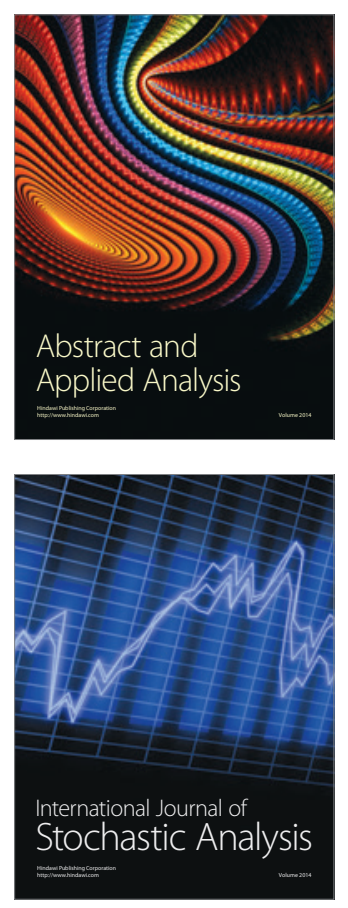

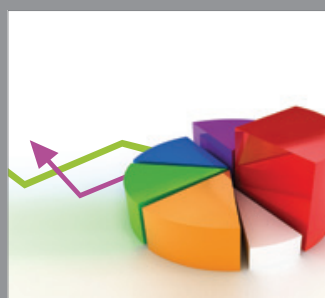

ournal of

Probability and Statistics

Promensencen
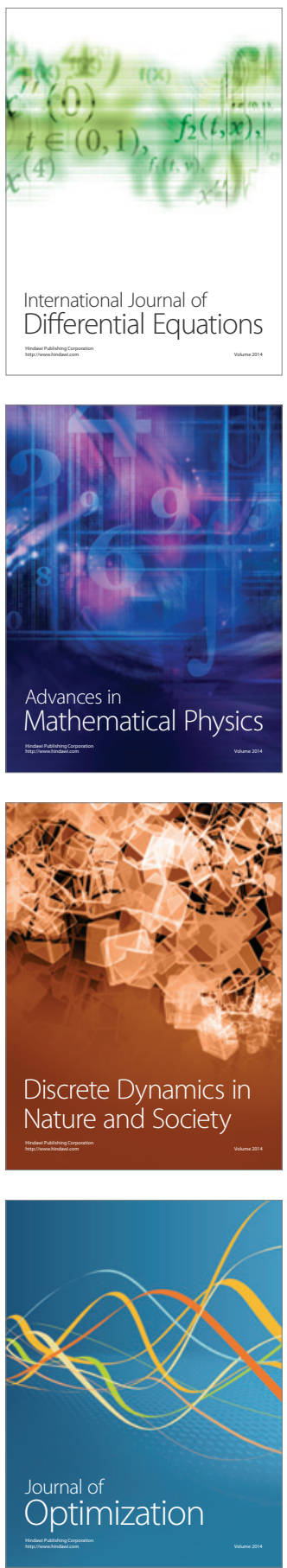